\title{
The potential roles of herpesvirus and cytomegalovirus in the exacerbation of pemphigus vulgaris
}

\author{
Fariba Mohammadi ${ }^{1}$, Zahra Khalili ${ }^{1}$, Sayed Mahdi Marashi ${ }^{2}$, Amirhoushang Ehsani ${ }^{1}$, \\ Maryam Daneshpazhooh ${ }^{1}$, Majid Teymoori-Rad ${ }^{2}$, Kamran Balighi ${ }^{1}$, Ahmad Nejati², \\ Shohreh Shahmahmoodi², Shima Izadidakhrabadi ${ }^{2}$, Hamidreza Mahmoudi ${ }^{1}$, \\ Pedarm Noormohammadpour ${ }^{1}$
}

\begin{abstract}
1 Autoimmune Bullous Disease Research Center, Department of Dermatology, Razi Hospital, Tehran University of Medical Sciences, Tehran, Iran

2 Virology Department, School of Public Health (SPH), Tehran University of Medical Sciences (TUMS), Tehran, Iran
\end{abstract}

Key words: pemphigus vulgaris, herpesvirus, cytomegalovirus

Citation: Mohammadi F, Khalili Z, Mahdi Marashi S, Ehsani A, Daneshpazhooh M, Teymoori-Rad M, Balighi K, Nejati A, Shahmahmoodi S, Izadidakhrabadi S, Mahmoudi H, Noormohammadpour P. The potential roles of herpesvirus and cytomegalovirus in the exacerbation of pemphigus vulgaris. Dermatol Pract Concept. 2018;8(4):262-271. DOI: https://doi.org/10.5826/dpc.0804a03

Received: March 19, 2018; Accepted: July 18, 2018; Published: October 31, 2018

Copyright: @2018 Mohammadi et al. This is an open-access article distributed under the terms of the Creative Commons Attribution License, which permits unrestricted use, distribution, and reproduction in any medium, provided the original author and source are credited.

Funding: This research was supported by Tehran University of Medical Sciences (TUMS), Grant Numbers 32855-30-03-95 and 30976101-04-94.

Competing interests: The authors have no conflicts of interest to disclose.

All authors have contributed significantly to this publication.

Corresponding author: Pedarm Noormohammadpour, MD, Autoimmune Bullous Disease Research Center, Razi Hospital, Vahdate-Eslami Square, 11996, Tehran, Iran. Email: normohamad@razi.tums.ac.ir

ABSTRACT Background: Among exogenous etiologies, the critical role of microbial agents such as herpesviruses (HSV1/2) and cytomegalovirus (CMV) in triggering and flaring autoimmune conditions such as pemphigus vulgaris (PV) has been recently discovered.

Objectives: The present study aimed to investigate the plausible role of these viruses in the exacerbation of PV using serological and molecular methods.

Patients/Methods: Sixty patients with PV (30 with relapse type and 30 with remission type) were recruited for the purpose of this case-control study. Skin, mucosal, and throat specimens were obtained and examined for viruses by reverse transcriptase polymerase chain reaction. To determine the immunoglobulin $\mathrm{G}(\mathrm{IgG})$ titer, enzyme-linked immunosorbent assay was used.

Results: Desmoglein1-specific IgG was positive in $56.7 \%$ of patients with the relapse form and in $20.0 \%$ of those with the remission form indicating a significant difference across the 2 groups $(P=0.003)$, but the rate of positivity for desmoglein3-specific $\operatorname{IgG}$ in the relapse and remission types was $76.7 \%$ and $63.3 \%$, respectively, with no significant difference $(P=0.260)$. There was no difference in the mean levels of HSV-IgG and CMV-IgG in the relapse and remission groups. HSV and 
ABSTRACT CMV positivity in PV patients was independent of the site of the samples. Using the multivariable linear regression model, the level of CMV-IgG in PV patients was directly affected by female sex and advanced ages.

Conclusions: Our study could not demonstrate the role of HSV1/2 and CMV as triggering factors for PV exacerbation. Further studies are needed to evaluate the potential role of these viruses in PV exacerbation especially considering demographic variables.

\section{Introduction}

Pemphigus disease is an autoimmune disease that is characterized by secretion of autoantibodies that act against surface glycoproteins of epithelial cells [1]. The immunological basis for pemphigus disease includes autoantibodies targeted against keratinocyte surface antigens desmoglein 1 and desmoglein3 (DSG1, DSG3) [2]. These proteins are primarily involved in intercellular cell-to-cell adhesion structures. Pemphigus vulgaris (PV) is a common type of pemphigus disease with painful blistering on the skin and mucous membranes [3]. Overall, the close link between autoimmune diseases and both endogenous (genetic) and exogenous (environmental) factors has been clearly understood. Exogenous factors include thiol drugs, physical trauma such as burn, ultraviolet exposure, X-ray, neoplasm, hormones and pregnancy, nutritional factors, and emotional stress [4-7].

Among exogenous etiologies, the critical role of microbial agents such as viruses in triggering and flaring autoimmune conditions has been recently discovered. In this regard, the causative role of herpesviruses (HSVs) as the most important human pathogens in the pathogenesis of PV has been recently suggested [8]. Thus, efforts have been made to clarify viral etiologies for PV and in this way the role of HSVs and cytomegalovirus (CMV) is taken into consideration [9,10]. The main common features of both pointed viruses include their ability to survive in the host body for a long time as well as activating periodically [11]. The human HSVs including herpesviruses 1 and 2 (HSV1/2) and CMV infect between 60\% and $90 \%$ of the adult population worldwide $[12,13]$. After primary infection, the virus establishes lifelong latency, with periodic reactivations that are effectively controlled by a robust immune response in most infected individuals despite the virus-producing proteins that interfere with adaptive and innate immunity. The activation of $\mathrm{T}$ cells is the important aspect of adaptive immunity. Tissue-resident memory (Trm) $\mathrm{T}$ cells are a subtype of memory lymphocytes that enter nonlymphoid tissues such as skin and become permanently established without recirculating. Local immune control of viruses can be mediated by Trm T cells through direct killing of infected cells and recruiting circulating memory CD8+ to the skin. Also, Trm T cells maintain HSV1 latency by secreting granzyme $\mathrm{B}$, which degrades the early protein ICP4 that is important in viral replication $[14,15]$.
Atypical HSV infections have been described in immunosuppressed patients [16]. Several studies have suggested the activation or exacerbation of pemphigus after HSV or CMV infections [17]. More recently, Japanese researchers have detected high levels of HSV in the saliva samples of PV patients [18]. In this regard, several studies have shown the benefits of adding antiviral therapies to an immunosuppressive therapy in recalcitrant disease $[19,20]$. Several studies also failed to detect herpesviruses in pemphigus patient specimens $[21,22]$, suggesting that viruses may have only a transient role for exacerbation of pemphigus disease, although yet to be further defined.

The number of patients with PV in Iran appears to be increasing, and little information is available with regard to the role of HSV1/2 and CMV in triggering PV among the Iranian population. The present study aimed to investigate the plausible role of these viruses (HSV1/2 and CMV) in the exacerbation of PV using serological and molecular methods.

\section{Materials and Methods}

\section{Disease Definitions in PV Patients}

PV patients were categorized as follows: relapse (development of more than 3 new lesions/month that do not heal within a week without treatment, or development of established lesions in a patient whose disease was controlled) and complete remission (absence of new or established lesions in a patient whose all-systemic therapy is discontinued for $\geq 2$ months, or while the patient is receiving minimal therapy) [23]. Scoring of disease was recorded based on the pemphigus disease area index.

\section{Study Population}

Sixty patients with PV (27 male, 33 female; mean age $46.3 \pm$ 12.7 years) were recruited for the purpose of this case-control study. Patients were selected among inpatients and outpatients who were referred to the pemphigus clinic of Razi Hospital (Tehran, Iran). The diagnosis of pemphigus had been confirmed by histology and direct immunofluorescence. Among patient subjects, 30 were in complete remission as in the control group, whereas the rest $(n=30)$ were in relapse as in the case group. None of the patients had overt labial herpetic lesions at the time of sampling. All study participants 
gave informed consent to provide samples for the study, which was approved by the Local Research Ethics Committee of Tehran University of Medical Sciences (approval number: IR.TUMS.MEDICINE.REC.1395.1017 and IR.TUMS. MEDICINE.REC.1395.2472).

\section{Sample Preparation}

For viral detection and determination of specific antibodies, we used blood, skin biopsy, and throat swab samples from patients. After patient consent forms were obtained, one skin specimen or mucosal specimens were obtained by punch biopsy $(3 \mathrm{~mm})$ and blood samples were taken from all patients. In addition, a sample from the throat was collected by swabbing. All samples were transferred in cold chain conditions to the virology laboratory. As soon as samples were received, preparation and storage were carried out. Sera (for serology) and buffy coat (for virus detection) were isolated from whole blood and stored at $-20^{\circ} \mathrm{C}$. DNA extraction from buffy coat, skin biopsy, and throat swab were performed using DNA Isolation Kit for Cells and Tissues according to the manufacturers' instructions (Roche, Berlin, Germany). After DNA extraction, it was eluted in $50 \mu \mathrm{L}$ of buffer and then adjusted to a definitive concentration of $500 \mathrm{ng} / \mu \mathrm{L}$.

\section{Virus-Specific Antibody Responses}

HSV1/2- and CMV-immunoglobulin G (IgG) antibodies were determined using enzyme-linked immunosorbent assay (ELISA). Quantification of IgG antibodies was determined by standard curve as RU/mL according to the manufacturer (values greater than $22 \mathrm{RU} / \mathrm{mL}$ were defined as seropositive).

\section{DSG-Specific IgG}

The levels of autoantibody to DSG1 and DSG3 were measured by ELISA. The cutoff values were $20 \mathrm{U} / \mathrm{mL}$ for both.

\section{Determination of Viral Load}

The absolute quantification of HSV1/2 and CMV-specific DNA was performed by real-time polymerase chain reaction (PCR) using the RealStar HSV PCR Kit 1.0 and RealStar CMV PCR Kit 1.0, respectively (Altona Diagnostics; Hamburg, Germany). All stages of the amplification of the viruses' specific target sequences for the detection and quantification of the amplified DNA were carried out according to the manufacturer's instructions. Ten microliters of the DNA extracted from samples in distilled water, and the 4 controls were included in each experiment as a template and a negative control and quantification standards, respectively. Moreover, we performed internal control to identify possible PCR's inhibitor and to confirm the reliability of all reagents of the kit used for each sample. Finally, the concentration of the samples was calculated in copies per microliter and copies per milliliter.

\section{Statistical Analysis}

The results were presented as mean \pm standard deviation for quantitative variables and were summarized by absolute frequencies and percentages for categorical variables. Normality of data was analyzed using the Kolmogorov-Smirnoff test. Categorical variables were compared using chi-squared test or Fisher's exact test when more than $20 \%$ of cells with an expected count of less than 5 were observed. Quantitative variables were also compared with t test or Mann-Whitney $\mathrm{U}$ test. The association between quantitative variables was assessed using the Pearson correlation test. To determine the main correlates of IgG antibodies against viruses, multivariable linear regression analysis was used. For the statistical analysis, the statistical software SPSS version 16.0 for Windows (SPSS Inc., Chicago, IL) was used. P values of 0.05 or less were considered statistically significant. We used GraphPad Prism software (GraphPad Software, La Jolla, CA) for plotting graphs.

\section{Results}

In total, 30 patients with the relapse type of PV and 30 patients with the remission type were assessed. The demographic and clinical features of patients are presented in Table 1. Comparison of the baseline characteristics of the 2 groups (Table 2) showed no difference in gender, mean age, subtypes of disease (mucous or mucocutaneous), or disease duration and oral medications. Regarding medical history, hypertension and hyperlipidemia were more prevalent in those with relapse PV than in the remission group, while no difference was revealed in other underlying comorbidities including diabetes mellitus, history of smoking, coronary artery disease, or thyroid abnormalities.

With regard to DSG-specific IgG positivity in the relapse and remission forms of PV, DSG1-specific IgG was positive in $56.7 \%$ of patients with the relapse form and in $20.0 \%$ of those with the remission form, indicating a significant difference across the 2 groups $(P=0.003)$, but the rate of positivity for DSG3-specific IgG in the relapse and remission types was $76.7 \%$ and $63.3 \%$, respectively, with no significant difference $(P=0.260)$. The mean HSV-IgG in the relapse and remission groups was $154.47 \pm 66.80$ and $151.17 \pm$ 62.73 , respectively, with no significant difference $(\mathrm{P}=$ 0.844). Moreover, the mean CMV-IgG in the subgroups was $130.30 \pm 47.88$ and $127.93 \pm 53.56$, respectively, with no meaningful difference ( $\mathrm{P}=0.857)$. There was no correlation between prednisolone dose and HSV-IgG $(P=0.373)$ or CMV-IgG (P = 0.647). As shown in Table 3, HSV and CMV positivity in PV patients was independent of the source of the samples. 
TABLE 1. Demographic and clinical characteristics of pemphigus vulgaris patients

\begin{tabular}{|c|c|c|c|c|c|c|c|c|c|c|}
\hline ID & Sex & $\begin{array}{c}\text { Age } \\
\text { (Years) }\end{array}$ & Type & $\begin{array}{c}\text { Duration } \\
\text { (Years) }\end{array}$ & Activation & $\begin{array}{l}\text { PDN Dose } \\
\text { (mg/d) }\end{array}$ & Drug History & DSG1 & DSG3 & PDAI \\
\hline P1 & $\mathrm{F}$ & 72 & MCPV & 2 & Relapse & 15 & Azathioprine & Positive & Positive & 94 \\
\hline $\mathrm{P} 2$ & $\mathrm{~F}$ & 41 & MPV & 2.5 & Remission & 5 & Azathioprine & Negative & Positive & 0 \\
\hline P3 & F & 47 & MCPV & 4 & Remission & 5 & $\begin{array}{c}\text { Mycophenolic acid, } \\
\text { Azathioprine }\end{array}$ & Negative & Positive & 0 \\
\hline P4 & $\mathrm{F}$ & 48 & MCPV & 1.5 & Remission & 5 & Mycophenolic acid & Negative & Positive & 0 \\
\hline P5 & $\mathrm{F}$ & 31 & MCPV & 2 & Remission & 5 & $\begin{array}{l}\text { Azathioprine, } \\
\text { rituximab }\end{array}$ & Positive & Positive & 0 \\
\hline P6 & $\mathrm{F}$ & 28 & MCPV & 5 & Relapse & 10 & $\begin{array}{c}\text { Mycophenolic acid, } \\
\text { Azathioprine }\end{array}$ & Positive & Positive & 12 \\
\hline P7 & $\mathrm{M}$ & 61 & MCPV & 4 & Remission & 5 & $\begin{array}{c}\text { Azathioprine, } \\
\text { Mycophenolic acid, } \\
\text { rituximab, Mtx }\end{array}$ & Negative & Positive & 0 \\
\hline P8 & $\mathrm{M}$ & 67 & MCPV & 10 & Relapse & 30 & $\begin{array}{l}\text { Cyclophosphamide, } \\
\text { Mycophenolic acid, } \\
\text { rituximab }\end{array}$ & Negative & Positive & 34 \\
\hline P9 & $\mathrm{F}$ & 63 & MCPV & 3.5 & Remission & 0 & $\begin{array}{l}\text { Mycophenolic acid } \\
\text { monotherapy }\end{array}$ & Negative & Negative & 0 \\
\hline P10 & $\mathrm{M}$ & 41 & MCPV & 3 & Remission & 0 & Mycophenolic acid & Positive & Negative & 0 \\
\hline P11 & $\mathrm{M}$ & 60 & MCPV & 2.5 & Remission & 5 & Azathioprine & Negative & Negative & 0 \\
\hline P12 & $\mathrm{M}$ & 45 & MCPV & 7 & Remission & 5 & $\begin{array}{c}\text { Mycophenolic acid, } \\
\text { Azathioprine }\end{array}$ & Negative & Negative & 0 \\
\hline P13 & $\mathrm{M}$ & 32 & MCPV & 10 & Remission & 7.5 & Azathioprine & Negative & Positive & 0 \\
\hline P14 & $\mathrm{M}$ & 41 & MCPV & 1.5 & Remission & 5 & $\begin{array}{l}\text { Azathioprine, } \\
\text { rituximab }\end{array}$ & Negative & Negative & 0 \\
\hline P15 & $\mathrm{M}$ & 38 & MCPV & 4 & Remission & 10 & $\begin{array}{c}\text { Azathioprine, } \\
\text { mycophenolic acid, } \\
\text { rituximab }\end{array}$ & Positive & Negative & 0 \\
\hline P16 & $\mathrm{M}$ & 55 & MPV & 2 & Remission & 7.5 & Azathioprine & Negative & Positive & 0 \\
\hline P17 & $\mathrm{M}$ & 65 & MCPV & 2 & Relapse & 5 & Azathioprine & Negative & Positive & 14 \\
\hline P18 & $\mathrm{F}$ & 45 & MCPV & 2 & Relapse & 10 & Azathioprine & Positive & Positive & 12 \\
\hline P19 & $\mathrm{F}$ & 58 & MCPV & 2 & Remission & 7.5 & Azathioprine & Negative & Positive & 0 \\
\hline P20 & $\mathrm{M}$ & 39 & MPV & 2 & Remission & 1.25 & Azathioprine & Negative & Negative & 0 \\
\hline $\mathrm{P} 21$ & $\mathrm{~F}$ & 45 & MPV & 2 & Relapse & 7.5 & Mycophenolic acid & Negative & Positive & 5 \\
\hline P22 & $\mathrm{M}$ & 21 & MCPV & 1 & Relapse & 15 & Azathioprine & Positive & Positive & 34 \\
\hline P23 & $\mathrm{F}$ & 65 & MCPV & 12 & Remission & 5 & None & Negative & Positive & 0 \\
\hline P24 & $\mathrm{F}$ & 45 & MCPV & 10 & Remission & 5 & Azathioprine & Negative & Positive & 0 \\
\hline $\mathrm{P} 25$ & $\mathrm{~F}$ & 33 & MCPV & 5 & Relapse & 0 & Azathioprine & & & 15 \\
\hline P26 & $\mathrm{M}$ & 44 & MPV & 19 & Remission & 7.5 & $\begin{array}{c}\text { Mycophenolic acid, } \\
\text { Azathioprine }\end{array}$ & Negative & Positive & 0 \\
\hline P27 & $\mathrm{F}$ & 54 & MCPV & 2.5 & Remission & 5 & $\begin{array}{l}\text { Mycophenolic acid, } \\
\text { rituximab }\end{array}$ & Positive & Positive & 0 \\
\hline P28 & $\mathrm{F}$ & 62 & MCPV & 3 & Remission & 2.5 & Azathioprine & Negative & Negative & 0 \\
\hline P29 & $\mathrm{F}$ & 55 & $\mathrm{MCPV}$ & 1.5 & Remission & 2.5 & $\begin{array}{c}\text { Mycophenolic acid, } \\
\text { Mtx }\end{array}$ & Negative & Negative & 0 \\
\hline P30 & $M$ & 38 & MCPV & 1.5 & Remission & 7.5 & Rituximab & Positive & Positive & 0 \\
\hline P31 & $\mathrm{F}$ & 67 & MCPV & 11 & Remission & 5 & $\begin{array}{c}\text { Azathioprine, } \\
\text { mycophenolic acid, } \\
\text { rituximab, Mtx }\end{array}$ & Negative & Positive & 0 \\
\hline P32 & $\mathrm{F}$ & 46 & MCPV & 0.5 & Remission & 7.5 & Rituximab & Negative & Negative & 0 \\
\hline
\end{tabular}

(Continued next page) 
TABLE 1. Demographic and clinical characteristics of pemphigus vulgaris patients (continued)

\begin{tabular}{|c|c|c|c|c|c|c|c|c|c|c|}
\hline ID & Sex & $\begin{array}{c}\text { Age } \\
\text { (Years) }\end{array}$ & Type & $\begin{array}{l}\text { Duration } \\
\text { (Years) }\end{array}$ & Activation & $\begin{array}{l}\text { PDN Dose } \\
(\mathrm{mg} / \mathrm{d})\end{array}$ & Drug History & DSG1 & DSG3 & PDAI \\
\hline P33 & $\mathrm{F}$ & 40 & MCPV & 12 & Remission & 10 & Azathioprine & Negative & Positive & 0 \\
\hline P34 & M & 40 & MCPV & 3 & Relapse & 25 & $\begin{array}{l}\text { Azathioprine, } \\
\text { rituximab }\end{array}$ & Negative & Positive & 5 \\
\hline P35 & M & 55 & MCPV & 6 & Remission & 7.5 & Azathioprine & Negative & Negative & 0 \\
\hline P36 & M & 35 & MCPV & 14 & Remission & 7.5 & $\begin{array}{l}\text { Mycophenolic acid, } \\
\text { Azathioprine, Mtx, } \\
\text { rituximab, IVIG }\end{array}$ & Negative & Positive & 0 \\
\hline P37 & M & 65 & MCPV & 5 & Relapse & 0 & $\begin{array}{c}\text { Mycophenolic acid, } \\
\text { Azathioprine }\end{array}$ & Positive & Negative & 23 \\
\hline P38 & $\mathrm{F}$ & 74 & MCPV & 2 & Relapse & 7.5 & Azathioprine & Positive & Positive & 24 \\
\hline P39 & $\mathrm{F}$ & 32 & MCPV & 2 & Relapse & 10 & Rituximab & Negative & Positive & 14 \\
\hline P40 & F & 39 & MCPV & 8 & Relapse & 2.5 & $\begin{array}{l}\text { Mycophenolic acid, } \\
\text { rituximab }\end{array}$ & Positive & Positive & 3 \\
\hline P41 & $\mathrm{F}$ & 36 & MCPV & 5 & Relapse & 5 & $\begin{array}{c}\text { Mycophenolic acid, } \\
\text { Azathioprine }\end{array}$ & Positive & Positive & 34 \\
\hline P42 & $\mathrm{F}$ & 54 & MCPV & 2 & Relapse & 5 & $\begin{array}{l}\text { Mycophenolic acid, } \\
\text { Azathioprine, Mtx }\end{array}$ & Positive & Positive & 12 \\
\hline P43 & $\mathrm{F}$ & 54 & MCPV & 6 & Relapse & 25 & $\begin{array}{c}\text { Rituximab, } \\
\text { mycophenolic acid }\end{array}$ & Negative & Positive & 11 \\
\hline P44 & $\mathrm{M}$ & 20 & MCPV & 1 & Relapse & 15 & Rituximab & Negative & Negative & 5 \\
\hline P45 & $\mathrm{F}$ & 42 & MPV & 1 & Remission & 2.5 & Rituximab & Negative & Positive & 0 \\
\hline P46 & $\mathrm{M}$ & 38 & MPV & 3 & Relapse & 15 & None & Negative & Positive & 27 \\
\hline P47 & $\mathrm{F}$ & 46 & MCPV & 15 & Relapse & 20 & $\begin{array}{l}\text { Mycophenolic acid, } \\
\text { Azathioprine, Mtx, } \\
\text { rituximab, IVIG, } \\
\text { cyclophosphamide }\end{array}$ & Positive & Positive & 0 \\
\hline P48 & $\mathrm{M}$ & 27 & MCPV & 1.5 & Relapse & 30 & $\begin{array}{c}\text { Mycophenolic acid, } \\
\text { Azathioprine }\end{array}$ & Positive & Positive & 0 \\
\hline P49 & M & 42 & MCPV & 0.5 & Relapse & 35 & None & Negative & Negative & 17 \\
\hline P50 & $\mathrm{F}$ & 34 & MPV & 1 & Relapse & 10 & $\begin{array}{l}\text { Mycophenolic acid, } \\
\text { Azathioprine, Mtx, } \\
\text { rituximab, IVIG, } \\
\text { cyclophosphamide }\end{array}$ & & & 10 \\
\hline P51 & $\mathrm{F}$ & 34 & MCPV & 1 & Relapse & 6.25 & Rituximab & Positive & Negative & 38 \\
\hline P52 & $\mathrm{F}$ & 37 & MCPV & 2 & Relapse & 25 & $\begin{array}{l}\text { Azathioprine, } \\
\text { rituximab }\end{array}$ & Positive & Positive & 19 \\
\hline P53 & $\mathrm{M}$ & 57 & MCPV & 3 & Relapse & 15 & Azathioprine & Positive & Positive & 84 \\
\hline P54 & $\mathrm{M}$ & 54 & MCPV & 5.5 & Relapse & 2.5 & $\begin{array}{l}\text { Azathioprine, } \\
\text { mycophenolic acid, } \\
\text { rituximab, Mtx }\end{array}$ & Positive & Negative & 12 \\
\hline P55 & $\mathrm{F}$ & 54 & MCPV & 2 & Relapse & 12.5 & Rituximab & Negative & Positive & 8 \\
\hline P56 & $\mathrm{F}$ & 48 & MCPV & 1 & Relapse & 10 & Azathioprine & Positive & Positive & 23 \\
\hline P57 & $\mathrm{F}$ & 60 & MCPV & 10 & Remission & 10 & Azathioprine & Negative & Positive & 0 \\
\hline P58 & $\mathrm{M}$ & 29 & MCPV & 3 & Relapse & 5 & $\begin{array}{l}\text { Mycophenolic acid, } \\
\text { rituximab }\end{array}$ & Positive & Positive & 14 \\
\hline P59 & $\mathrm{M}$ & 45 & MCPV & 1 & Relapse & 15 & Azathioprine & Positive & Positive & 21 \\
\hline P60 & $\mathrm{F}$ & 39 & MCPV & 1.5 & Relapse & 35 & $\begin{array}{l}\text { Mycophenolic acid, } \\
\text { Azathioprine }\end{array}$ & Negative & Positive & 15 \\
\hline
\end{tabular}

Abbreviations: Mtx, methotrexate; DSG, desmoglein; MCPV, mucocutaneous PV; MPV, mucous PV; PDN dose, oral prednisolone dose at the time of the study; PDAI, pemphigus disease area index 
TABLE 2. Comparing baseline characteristics between the patients with relapse and those in remission

\begin{tabular}{|c|c|c|c|}
\hline & Relapse Type $(n=30)$ & Remission Type $(n=30)$ & P Value \\
\hline Male & $12(40.0)$ & $14(46.7)$ & 0.602 \\
\hline Mean Age, Years $( \pm$ SD $)$ & $44.90 \pm 14.30$ & $47.83 \pm 11.04$ & 0.377 \\
\hline Disease Duration, Years $( \pm \mathrm{SD})$ & $3.42 \pm 3.14$ & $5.23 \pm 4.75$ & 0.086 \\
\hline \multicolumn{4}{|l|}{ Medical History } \\
\hline History of hypertension & $9(30.0)$ & $2(6.7)$ & 0.020 \\
\hline History of hyperlipidemia & $5(16.7)$ & $0(0.0)$ & 0.050 \\
\hline History of diabetes mellitus & $5(16.7)$ & $2(6.7)$ & 0.424 \\
\hline History of smoking & $1(3.3)$ & $1(3.3)$ & 1.000 \\
\hline History of coronary disease & $2(6.7)$ & $1(3.3)$ & 0.998 \\
\hline History of AVN & $2(6.7)$ & $0(0.0)$ & 0.492 \\
\hline History of hypothyroidism & $2(6.7)$ & $0(0.0)$ & 0.492 \\
\hline Form of Disease & & & 0.706 \\
\hline Mucous & $3(10.0)$ & $5(16.7)$ & \\
\hline Mucocutaneous & $27(90.0)$ & $25(83.3)$ & \\
\hline \multicolumn{4}{|l|}{ Medication } \\
\hline Azathioprine & $19(63.3)$ & $20(66.7)$ & 0.787 \\
\hline Mycophenolic acid & $13(43.3)$ & $13(43.3)$ & 1.000 \\
\hline Rituximab & $12(40.0)$ & $10(33.3)$ & 0.592 \\
\hline Methotrexate & $4(13.3)$ & $4(13.3)$ & 1.000 \\
\hline IVIG & $2(6.7)$ & $1(3.3)$ & 0.999 \\
\hline Cyclophosphamide & $3(10.0)$ & $0(0.0)$ & 0.237 \\
\hline
\end{tabular}

Unless noted otherwise, values represent number (percent).

TABLE 3. Comparison of HSV and CMV detection based on reverse transcriptase polymerase chain reaction techniques according to the source of samples between the patients with relapse and those in remission

\begin{tabular}{|l|c|c|c|}
\hline & Relapse Type $(\mathbf{n}=\mathbf{3 0})$ & Remission Type $(\mathbf{n}=\mathbf{3 0})$ & P Value \\
\hline HSV & & & 0.998 \\
\hline Skin & $1(3.3)$ & $0(0.0)$ & 0.898 \\
\hline ThMC & $1(3.3)$ & $2(6.7)$ & 1.000 \\
\hline CMV & $0(0.0)$ & $0(0.0)$ & 0.237 \\
\hline Skin & $3(10.0)$ & $0(0.0)$ & 0.052 \\
\hline PBMC & $5(16.7)$ & $0(0.0)$ & 0.237 \\
\hline Throat & $3(10.0)$ & $0(0.0)$ & \\
\hline
\end{tabular}

Values represent number (percent).

Abbreviation: PBMC, peripheral blood mononuclear cell.

As indicated in Table 4 and Figure 1 and using the multivariable linear regression model, the level of HSV-IgG was not associated with the baseline characteristics including demographics, underlying comorbidities, disease duration, and form of PV. However, as shown in Table 5 and Figure 2, the level of CMV-IgG in PV patients was affected by the gender and age factors. The mean CMV-IgG was significantly higher in female than in male patients suffering from PV (142.21 \pm
46.60 vs $112.00 \pm 50.86, \mathrm{P}=0.020)$. Also, a direct correlation was revealed between patients' age and the level of CMV-IgG $(\mathrm{r}$ coefficient $=0.404, \mathrm{P}=0.001)$.

\section{Discussion}

Several studies have investigated the relationship between viral infections and the initiation or exacerbation of autoimmune 
TABLE 4. Multivariable linear regression model to assess the main correlates of HSV-IgG antibody in patients with pemphigus vulgaris

\begin{tabular}{|c|c|c|c|c|c|}
\hline \multirow[b]{2}{*}{ (Constant) } & \multicolumn{2}{|c|}{ Unstandardized Coefficients } & \multirow{2}{*}{$\begin{array}{c}\begin{array}{c}\text { Standardized } \\
\text { Coefficients }\end{array} \\
\text { Beta }\end{array}$} & \multirow{2}{*}{$\mathbf{t}$} & \multirow{2}{*}{ P Value } \\
\hline & B & Std. Error & & & \\
\hline Sex & -35.727 & 164.984 & & -0.217 & 0.829 \\
\hline Age & -3.982 & 18.674 & -0.031 & -0.213 & 0.832 \\
\hline Type & 0.492 & 0.809 & 0.098 & 0.608 & 0.546 \\
\hline Duration & -10.558 & 26.124 & -0.056 & -0.404 & 0.688 \\
\hline Form & 0.825 & 2.345 & 0.053 & 0.352 & 0.726 \\
\hline Hypertension & -15.421 & 19.720 & -0.121 & -0.782 & 0.438 \\
\hline Hyperlipidemia & 0.185 & 34.852 & 0.001 & 0.005 & 0.996 \\
\hline Diabetes & 58.505 & 46.066 & 0.254 & 1.270 & 0.210 \\
\hline Coronary Disease & -14.900 & 33.394 & -0.075 & -0.446 & 0.657 \\
\hline Smoking & -2.827 & 45.347 & -0.010 & -0.062 & 0.951 \\
\hline (Constant) & 67.297 & 54.454 & 0.190 & 1.236 & 0.222 \\
\hline
\end{tabular}

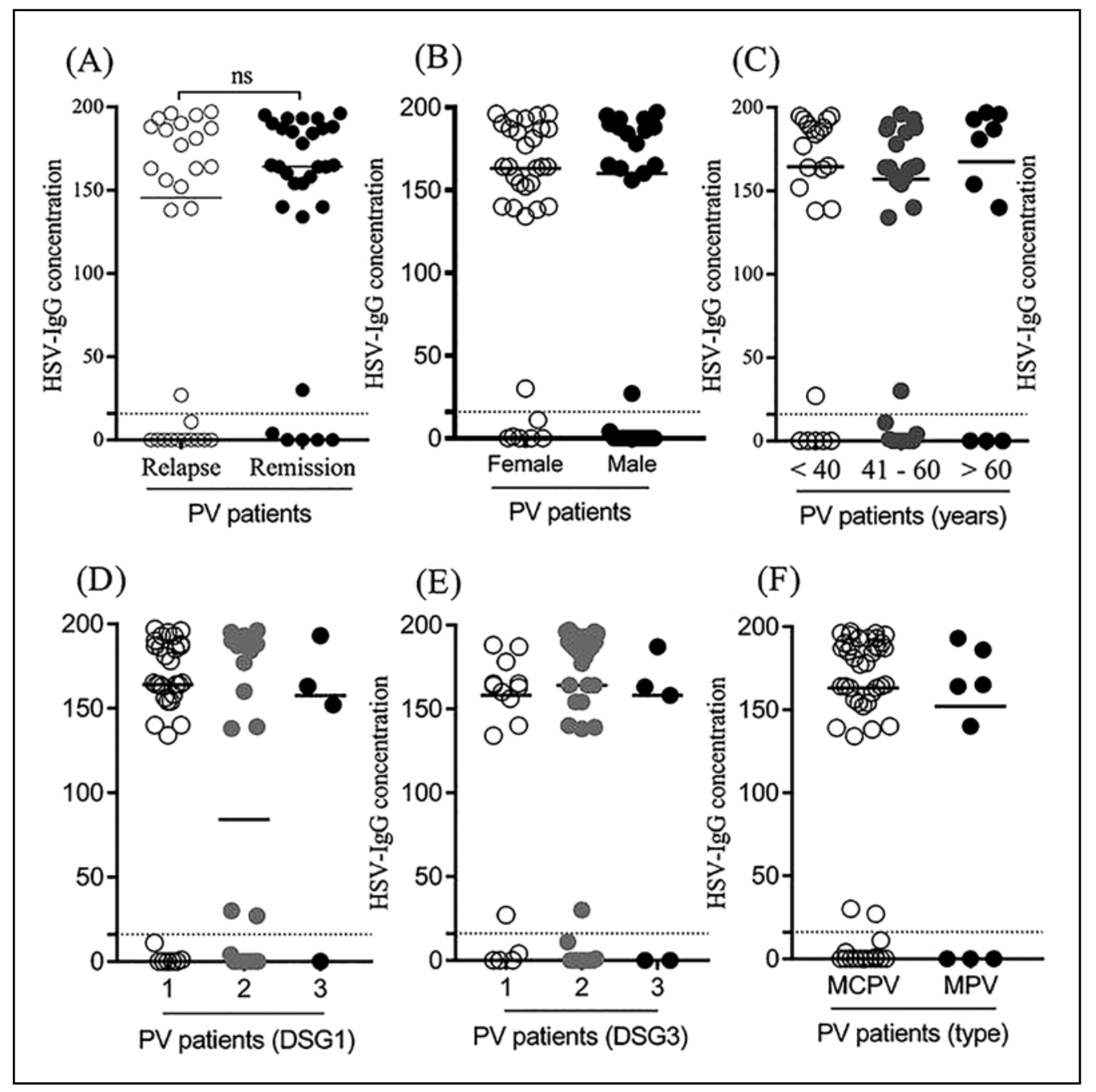

Figure 1. Level of HSV-IgG antibody (cut-off value: $22 \mathrm{RU} / \mathrm{mL}$ ) according to baseline variables. 
TABLE 5. Multivariable linear regression model to assess the main correlates of CMV-IgG antibody in patients with pemphigus vulgaris

\begin{tabular}{|l|c|c|c|c|c|} 
& \multicolumn{2}{|c|}{ Unstandardized Coefficients } & $\begin{array}{c}\text { Standardized } \\
\text { Coefficients }\end{array}$ & t & P Value \\
\cline { 1 - 3 } \multicolumn{1}{|c|}{ (Constant) } & B & Std. Error & Beta & & 0.585 \\
\hline Sex & -62.825 & 114.201 & & -0.550 & 0.033 \\
\hline Age & 28.334 & 12.926 & 0.281 & 2.192 & 0.038 \\
\hline Type & 1.196 & 0.560 & 0.303 & 2.135 & 0.190 \\
\hline Duration & 24.026 & 18.083 & 0.163 & 1.329 & 0.752 \\
\hline Form & -0.515 & 1.623 & -0.042 & -0.317 & 0.870 \\
\hline Hypertension & -2.244 & 13.650 & -0.022 & -0.164 & 0.558 \\
\hline hyperlipidemia & -14.238 & 24.124 & -0.110 & -0.590 & 0.693 \\
\hline Diabetes & 12.675 & 31.886 & 0.070 & 0.397 & 0.131 \\
\hline Coronary dis. & 35.510 & 23.115 & 0.228 & 1.536 & 0.146 \\
\hline Smoking & -46.372 & 31.389 & -0.202 & -1.477 & 0.298 \\
\hline (Constant) & 39.650 & 37.693 & 0.142 & 1.052 & 0.50 \\
\hline
\end{tabular}

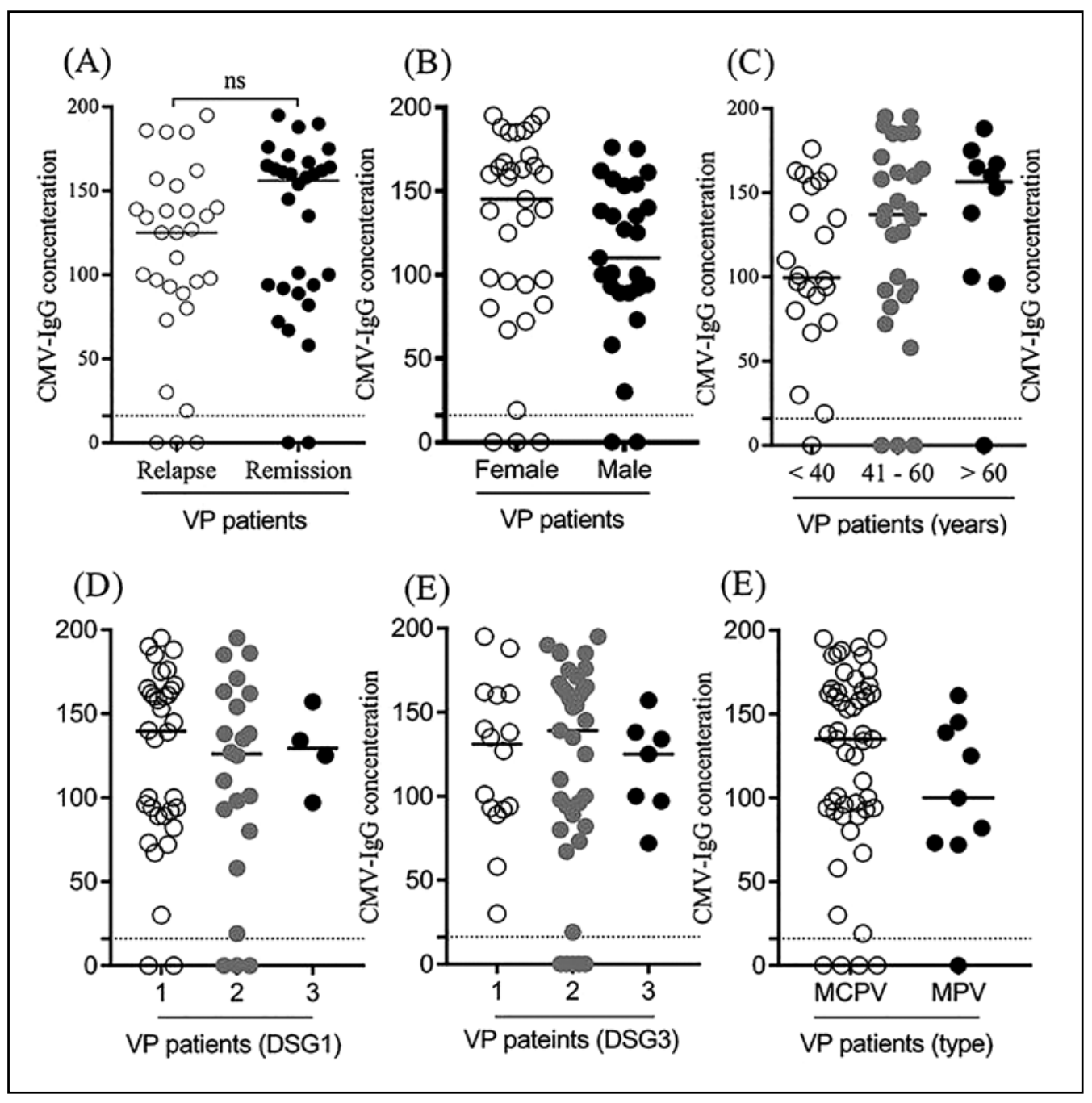

Figure 2. Level of CMV-IgG antibody (cut-off value: $22 \mathrm{RU} / \mathrm{mL}$ ) according to baseline variables. 
diseases such as systemic lupus erythematosus, rheumatoid arthritis, multiple sclerosis, and diabetes mellitus [24]. In this regard, efforts have been made to detect viral pathogens in blood samples and skin specimens of patients with pemphigus by techniques such as immunochemistry and PCR for DNA particles. The association between onset or reactivation of pemphigus disease and viral infections may be causal, or as a result of drug-induced immunosuppression which can increase the likelihood of viral infections, or established on the pathogenic connection between viral infection and immune dysregulation leading to autoimmunity. The mechanism of viral induction of autoimmunity can be explained in several ways, including molecular mimicry (due to cross-reactions between pathogen-derived epitopes and self-derived epitopes); bystander activation (due to activation of preexisting autoreactive $\mathrm{T}$ cells as a result of the inflammatory environment); epitope spreading (when antigen-presenting cells recognize self-antigens which were not initially directed by the immune system); polyclonal activation (due to infection of $\mathrm{B}$ cells by lymphocytotropic viruses which lead to B cell proliferation and increased antibody production); and viral superantigens (encoded by some viruses, are able to activate polyclonal $\mathrm{T}$ cells) [25]. Molecular mimicry is possibly the most acceptable explanation for the association of viral infections and pemphigus. Further explanation is that viruses upregulate production of interferon and interleukins. High levels of interferon gamma lead to increased expression of HLA2 on keratinocytes, making the pemphigus antigen structural site active. Also, overproduction of IL4 and IL10 causes a shift of TH1 to TH2 response which potentiates antibody response [26,27].

The role of viruses in the pathogenesis of pemphigus has been assessed in some previous studies. 'The importance of viral infections as an exacerbating factor of PV is neglected by some clinicians. Concerning this, in our current case-control study, we evaluated the probable role of HSV1/2 and CMV in the exacerbation of PV. In our study, a significantly higher number of PV patients (regardless of disease activity) were positive for HSV- and CMV-IgG antibodies, similar to the studies by Tufano et al and Sagi et al $[28,29]$. This may show a history of HSV and CMV exposure in most patients and may indicate more frequent recrudescence of latent viruses [30]. A significant increase in IgG antibody levels, discovered by comparing relapse and remission samples, indicates an active infection. According to a general consensus, relatively higher IgG levels are associated with a higher probability of having an active viral infection and relatively lower IgG levels represent a previous viral infection rather than an active viral infection. In this regard, Senger et al showed higher levels of HSV1 antibody in patients in the active phase of disease than in remission patients [31]. In contrast to our study, there was no significant difference in antibody levels between relapse and remission patients. In the studies of Marzano et al and Rah-
mati-Roodsari et al, similar to our study, only 1 patient had a positive HSV PCR result for skin samples [32,33]. These values are less than the findings of the study by Tufano et al, reporting positive HSV PCR in 5 of 7 skin specimens [29]. Other PCR results show evidence of HSV1 infection in $10 \%$ of all blood samples and CMV infection in $16.6 \%$ of all blood samples, $10 \%$ of all skin samples, and $10 \%$ of all throat samples. On the other hand, several studies failed to detect herpes DNA viruses in pemphigus patient specimens $[26,27]$. Senger et al showed the role of HSV1 in exacerbation of pemphigus by using the ELISA technique [31]. However, we found no correlation between HSV and CMV PCR results or IgG antibody levels and disease activity (relapse or remission). These findings cannot rule out the role of these viruses in exacerbation of the disease; improvement in some patients, especially those with the mucosal form, after receiving antiviral therapy as well as exacerbation of pemphigus after HSV infection are clinical evidence of the possible role of viruses in disease exacerbation [22]. Therefore, the role of viruses in exacerbation of pemphigus remains a hypothesis and requires further study.

Our study is unique for several reasons. First, we used both ELISA and PCR techniques to find evidence of viruses, whereas most previous studies used only one technique. Second, the samples were sourced from several sites including skin, throat, and blood. Third, we evaluated virus status in relapse and remission PV patients to determine the role of viruses in the exacerbation of the disease, whereas most previous studies examined the status of viruses in newly diagnosed patients and healthy controls to determine the etiological role of viruses. However, this study had some limitations. We could not evaluate other types of pemphigus disease due to their lower prevalence. Because of high costs of the techniques, we were able to investigate only the role of HSV1/2 and CMV in the exacerbation of disease.

\section{Conclusions}

In conclusion, our study could not demonstrate the role of HSV1/2 and CMV as triggering factors for PV exacerbation. Further studies are needed to evaluate the potential role of these viruses in PV exacerbation.

\section{References}

1. Kasperkiewicz M, Ellebrecht CT, Takahashi H, et al. Pemphigus. Nat Rev Dis Primers. 2017;3:17026.

2. Spindler V, Waschke J. Pemphigus: a disease of desmosome dysfunction caused by multiple mechanisms. Front Immunol. 2018;9:136.

3. Kridin K. Pemphigus group: overview, epidemiology, mortality, and comorbidities. Immunol Res. 2018;66(2):255-270.

4. Tur E, Brenner S. Contributing exogenous factors in pemphigus. Int J Dermatol. 1997;36(12):888-893. 
5. Paolino G, Didona D, Magliulo G, et al. Paraneoplastic pemphigus: insight into the autoimmune pathogenesis, clinical features and therapy. Int J Mol Sci. 2017;18(12):E2532.

6. Ruocco E, Wolf R, Ruocco V, Brunetti G, Romano F, Lo Schiavo A. Pemphigus: associations and management guidelines: facts and controversies. Clin Dermatol. 2013;31(4):382-390.

7. Tavakolpour S. Pemphigus trigger factors: special focus on pemphigus vulgaris and pemphigus foliaceus. Arch Dermatol Res. 2018;310(2):95-106.

8. Ruocco E, Ruocco V, Lo Schiavo A, Brunetti G, Wolf R. Viruses and pemphigus: an intriguing never-ending story. Dermatology. 2014;229(4):310-315.

9. Brandão ML, Fernandes NC, Batista DP, Santos N. Refractory pemphigus vulgaris associated with herpes infection: case report and review. Rev Inst Med Trop Sao Paulo. 2011;53(2):113-117.

10. Oliveira-Batista DP, Janini ME, Fernandes NC, Santos N. Laboratory diagnosis of herpesvirus infections in patients with pemphigus vulgaris lesions. Intervirology. 2013;56(4):231-236.

11. Marzano AV, Tourlaki A, Merlo V, Spinelli D, Venegoni L, Crosti C. Herpes simplex virus infection and pemphigus. Int J Immunopathol Pharmacol. 2009;22(3):781-786.

12. Looker KJ, Magaret AS, Turner KM, Vickerman P, Gottlieb SL, Newman LM. Global estimates of prevalent and incident herpes simplex virus type 2 infections in 2012. PLoS One. 2015;10(1):e114989.

13. Swanson EC, Schleiss MR. Congenital cytomegalovirus infection: new prospects for prevention and therapy. Pediatr Clin North Am. 2013;60(2):335-349.

14. Schenkel JM, Masopust D. Tissue-resident memory T cells. Immunity. 2014;41(6):886-897.

15. Ariotti S, Hogenbirk MA, Dijkgraaf FE, et al. T cell memory. Skin-resident memory CD8(+) T cells trigger a state of tissue-wide pathogen alert. Science. 2014;346(6205):101-105.

16. Desai DV, Kulkarni SS. Herpes simplex virus: the interplay between HSV, host, and HIV-1. Viral Immunol. 2015;28(10):546-555.

17. Brandão ML, Fernandes NC, Batista DP, Santos N. Refractory pemphigus vulgaris associated with herpes infection: case report and review. Rev Inst Med Trop Sao Paulo. 2011;53(2):113-117.

18. Takahashi I, Kobayashi TK, Suzuki H, Nakamura S, Tezuka F. Coexistence of pemphigus vulgaris and herpes simplex virus infection in oral mucosa diagnosed by cytology, immunohistochemistry, and polymerase chain reaction. Diagn Cytopathol. 1998;19(6):446-450.

19. Machado ARDSR, La Serra L, Turatti A, Machado AM, Roselino AM. Herpes simplex virus 1 and cytomegalovirus are associated with pemphigus vulgaris but not with pemphigus foliaceus disease. Exp Dermatol. 2017;26(10):966-968.
20. Oliveira-Batista DP, Janini ME, Fernandes NC, Santos N. Laboratory diagnosis of herpesvirus infections in patients with pemphigus vulgaris lesions. Intervirology. 2013;56(4):231-236.

21. Cohen SS, Blauvelt A, Weinstein MD, Herndier BG, Anhalt GJ. No evidence of human herpesvirus 8 infection in patients with paraneoplastic pemphigus, pemphigus vulgaris, or pemphigus foliaceus. J Invest Dermatol. 1998;111(5):781-783.

22. Esmaili N, Hallaji Z, Abedini R, Soori T, Mortazavi H, ChamsDavatchi C. Pemphigus vulgaris and herpesviruses: is there any relationship? Int J Dermatol. 2010;49(11):1261-1265.

23. Murrell DF, Dick S, Ahmed AR, et al. Consensus statement on definitions of disease, end points, and therapeutic response for pemphigus. J Am Acad Dermatol. 2008;58(6):1043-1046.

24. Ercolini AM, Miller SD. The role of infections in autoimmune disease. Clin Exp Immunol. 2009;155(1):1-15.

25. Lossius A, Johansen JN, Torkildsen $\varnothing$, Vartdal F, Holmoy T. Epstein-Barr virus in systemic lupus erythematosus, rheumatoid arthritis and multiple sclerosis: association and causation. Viruses. 2012;4(12):3701-3730.

26. Ruocco E, Ruocco V, Lo Schiavo A, Brunetti G, Wolf R. Viruses and pemphigus: an intriguing never-ending story. Dermatology. 2014;229(4):310-315.

27. Vercelli D, Jabara HH, Lauener RP, Geha RS. IL-4 inhibits the synthesis of IFN-gamma and induces the synthesis of IgE in human mixed lymphocyte cultures. J Immunol. 1990;144(2):570-573.

28. Sagi L, Baum S, Agmon-Levin N, et al. Autoimmune bullous diseases: the spectrum of infectious agent antibodies and review of the literature. Autoimmun Rev. 2011;10(9):527-535.

29. Tufano MA, Baroni A, Buommino E, Ruocco E, Lombardi ML, Ruocco V. Detection of herpesvirus DNA in peripheral blood mononuclear cells and skin lesions of patients with pemphigus by polymerase chain reaction. Br J Dermatol. 1999;141(6):10331039.

30. Hashido M, Kawana T. Herpes simplex virus-specific IgM, IgA and $\operatorname{IgG}$ subclass antibody responses in primary and nonprimary genital herpes patients. Microbiol Immunol. 1997;41(5):415-420.

31. Senger P, Abidi N, Lin DM, Seiffert-Sinha K, Sinha AA. Positive correlation of anti-herpes simplex type I virus antibody levels with pemphigus vulgaris disease status and activity in a large patient cohort. Eur J Dermatol. 2017;27(2):132-138.

32. Marzano AV, Tourlaki A, Merlo V, Spinelli D, Venegoni L, Crosti C. Herpes simplex virus infection and pemphigus. Int J Immunopathol Pharmacol. 2009;22(3):781-786.

33. Rahmati-Roodsari M, Rahmdar SR, Alfaragi M, Saeedi M, Rahmati Roodsari S, Sajadi Nia RS. Association of HSV1/2 infection and pemphigus disease. Arch Pediatr Infect Dis. 2014;2(2):207210. 